\title{
Rendimiento productivo e integridad ósea de pollos de carne en respuesta a suplementación dietaria con cuatro fuentes de fitasa comercial
}

\author{
PerformanCe AND BONE INTEGRITY OF BROILERS IN RESPONSE TO DIETARY \\ SUPPLEMENTATION WITH FOUR SOURCES OF COMMERCIAL PHYTASES
}

José Aguilar V. ${ }^{1,2,3}$, Otto Zea M. ${ }^{1}$, Carlos Vílchez P. ${ }^{1,4}$

\section{Resumen}

\begin{abstract}
El objetivo del presente ensayo fue determinar el efecto de cuatro fuentes de fitasas comerciales sobre el rendimiento productivo y la integridad ósea de pollos de carne a los 28 días de edad. Se utilizaron 300 pollos BB machos de la Línea Cobb 500 distribuidos al azar en 30 corrales en piso ( 10 aves por corral), alimentados durante 28 días con uno de los siguientes tratamientos: T1, Dieta normal en fósforo (DNP); T2, Dieta deficiente en fósforo (DDP); T3, DDP + Fitasa A (100 g/t); T4, DDP + Fitasa B (100 g/t); T5, DDP + Fitasa C $(50 \mathrm{~g} / \mathrm{t})$ y T6, DDP + Fitasa D $(50 \mathrm{~g} / \mathrm{t})$. El suministro de alimento y agua fue $\mathrm{ad}$ libitum. El peso vivo, ganancia de peso, consumo de alimento, conversión alimentaria y mortalidad se registraron semanalmente. A los 28 días de edad, 20 aves por tratamiento fueron sacrificados para determinar el rendimiento de carcasa, rendimiento de piernas, peso de tibias y, posteriormente, contenidos de humedad y ceniza en tibias. Los resultados mostraron que el comportamiento productivo de los pollos de carne alimentados con niveles normales de fósforo ( $\mathrm{T} 1)$ fueron significativamente superiores $(\mathrm{p}<0.05)$ al resto de tratamientos; asimismo, los tratamientos T3 y T5 y los tratamientos T4 y T6 presentaron respuestas similares, mientras que T2 (nivel deficiente de fósforo), mostró rendimientos menores. Las tibias de las aves de los tratamientos, con excepción del T2 $(\mathrm{p}<0.05)$, mostraron niveles adecuados de cenizas. Se concluye que las respuestas productivas de las aves que recibieron la dieta deficiente en fósforo con la adición de fitasas fueron menores que el nivel de respuesta de las aves alimentadas con la dieta normal en fósforo.
\end{abstract}

Palabras clave: fitasa; pollo de carne; rendimiento productivo; integridad ósea

\footnotetext{
${ }^{1}$ Departamento Académico de Nutrición, Facultad de Zootecnia, Universidad Nacional Agraria La Molina, Lima, Perú

${ }^{2}$ Departamento Académico de Nutrición, Universidad Nacional Autónoma de Alto Amazonas, Yurimaguas, Perú

${ }^{3}$ E-mail:viravas@outlook.com

${ }^{4}$ E-mail: cvilchezp@lamolina.edu.pe
}

Recibido: 27 de febrero de 2017

Aceptado para publicación: 29 de septiembre de 2017 
The aim of this study was to determine the effects of four sources of commercial phytases on the performance and bone integrity of broilers from 1 to 28 days of age. Three hundred 1-day old Cobb 500 male chickens were distributed in 30 pens (10 birds per pen) and fed with the experimental diets for 28 days. The treatments were: T1, diet with adequate phosphorus (PAD); T2, phosphorus deficient diet (PDD); T3, PDD + phytase A $(100 \mathrm{~g} / \mathrm{t}) ; \mathrm{T} 4, \mathrm{PDD}+$ phytase B $(100 \mathrm{~g} / \mathrm{t}) ; \mathrm{T} 5, \mathrm{PDD}+$ phytase C $(50 \mathrm{~g} / \mathrm{t})$ and T6, PDD + phytase D $(50 \mathrm{~g} / \mathrm{t})$. Feeds and water were provided ad libitum. Body weight, body weight gain, feed consumption, feed conversion, and mortality were recorded weekly. At 28 days of age, 20 chicks per treatment were slaughtered to determine carcass and leg yield and tibia weight, plus bone moisture and ash content in the tibia. The results showed that the productive performance of broilers fed diets with normal levels of phosphorus $(\mathrm{T} 1)$ were significantly higher $(\mathrm{p}<0.05)$ than chicks in the other treatments. Similarly, treatments T3 and T5 and treatments T4 and T6 presented similar responses, while T2 (deficient level of phosphorus) showed lower yields. Bone ash in all treatments, except for T2 $(\mathrm{p}<0.05)$, showed adequate levels. It was concluded that the productive responses of the birds that received diets deficient in phosphorus with the addition of phytase were lower than the level of response of the birds fed the diet with adequate level of phosphorus.

Key words: phytase; broilers; productive performance; bone integrity

\section{INTRODUCCIÓN}

El rendimiento productivo en pollos de carne no siempre alcanza niveles esperados de producción, debido a ciertos factores que dificultan el aprovechamiento de nutrientes esenciales. El fósforo es un mineral importante en el organismo animal, responsable de la formación y mantenimiento del hueso, especialmente durante el crecimiento y desarrollo esquelético de los pollos (Assuena et al., 2009; Shaw et al., 2010).

El fósforo se encuentra mayormente ligado a complejos que dificultan su aprovechamiento, especialmente el ácido fítico, el cual se desempeña como un factor antinutricional. Las aves carecen de las enzimas con capacidad para hidrolizar el ácido fítico y liberar los elementos ligados (Harland y Morris, 1995). De esta manera, la baja utilización del fósforo presente en granos $\mathrm{y}$ oleaginosas se traduce en pérdida de valiosos nutrientes, contribuyendo, además, al incremento en la excreción de este mineral al medioambiente (Nair y Duvnjak, 1991; Penn, 2004; Angel et al., 2006).

La biodisponibilidad del fósforo puede ser incrementada mediante el empleo de enzimas fitasas, producidas por microorganismos, que se caracterizan por aumentar la disponibilidad de fósforo en los alimentos que están en forma de fitato (Woyengo et al., 2010). Actualmente, en el mercado nacional se disponen de diversos tipos de fitasas microbiales, cada una con relativa efectividad para liberar fósforo fítico; sin embargo, no existen suficientes trabajos de investigación, bajo condiciones controladas, sobre el rendimiento productivo y desarrollo óseo que comparen la efectividad de estos productos y permitan dilucidar su desempeño y eficacia en la nutrición de pollos de carne (Godoy et al., 2002; Hussein, 2006). 
Es por esto que el presente trabajo de investigación tuvo como objetivo evaluar el efecto de cuatro fuentes de fitasas comerciales sobre el comportamiento productivo e integridad ósea de pollos de carne de 1 a 28 días de edad, alimentados con dietas deficientes en fósforo.

\section{Materiales Y Métodos}

El estudio se llevó a cabo en la Unidad Experimental de Avicultura de la Facultad de Zootecnia de la Universidad Nacional Agraria La Molina (UNALM), Lima, Perú. Se emplearon 300 pollos BB machos de un día de edad, de la línea Cobb 500, distribuidos al azar en seis tratamientos $(\mathrm{n}=50)$, con 5 unidades experimentales por tratamiento de 10 aves cada una. La prueba fue realizada en un galpón para pollos distribuidos en corrales en piso de $1.5 \mathrm{~m}$ de largo por $0.7 \mathrm{~m}$ de ancho y $0.4 \mathrm{~m}$ de alto. Los corrales presentaban comederos y bebederos de tipo plástico durante los seis primeros días, y comederos y bebederos lineales automáticos en el resto del estudio. La calefacción fue provista por un sistema de campanas calefactoras. El tiempo de crianza fue de 28 días.

Los tratamientos evaluados fueron los siguientes: T1, Dieta normal en fósforo (DNP); T2, Dieta deficiente en fósforo (DDP); T3, DDP + fitasa A (100 g/t); T4, DDP + fitasa B $(100 \mathrm{~g} / \mathrm{t}) ; \mathrm{T} 5, \mathrm{DDP}+$ fitasa $\mathrm{C}(50 \mathrm{~g} / \mathrm{t})$ y T6, DDP + fitasa D $(50 \mathrm{~g} / \mathrm{t})$. Las marcas comerciales de las fitasas A, B, C y D fueron Abtusa (A/B Tecnologies International), Lumiphytase (Lumis Enzymes), Natuzyme-QP10 (Bioproton) y Challenge (Beijing Challenge Bio-technology), respectivamente. Las cantidades de las fitasas comerciales fueron determinadas en función de las recomendaciones de la marca comercial.

Las especificaciones nutricionales estuvieron de acuerdo a la guía nutricional para pollos de carne de la línea Cobb 500 (CobbVantress, 2012). Para T1 (dieta nivel normal en $\mathrm{P})$ se utilizó el requerimiento óptimo $(0.45 \%$ de $\mathrm{P}$ disponible [Pd]) y para T2 (dieta deficiente en $\mathrm{P}$ ) se utilizó el requerimiento mínimo $(0.25 \%$ de $\mathrm{Pd})$. El análisis proximal de las dietas y los niveles de fósforo de los ingredientes se desarrollaron en el Laboratorio de Evaluación Nutricional de Alimentos (LENA) de la UNALM. En el Cuadro 1 se muestra la composición, los valores nutricionales calculados y de los nutrientes de las dietas experimentales.

Se determinó la ganancia de peso, el consumo de alimento y la conversión alimenticia acumulada. Al final del estudio se determinó el índice de eficiencia productivo europeo, el rendimiento de carcasa, el rendimiento de piernas y el porcentaje de mortalidad. El peso vivo y el consumo de alimento se midieron utilizando una balanza digital de plataforma con precisión de $0.01 \mathrm{~g}$. El consumo voluntario de alimento se determinó restando la cantidad de alimento ofrecido de la suma del residuo del alimento y su desperdicio. La conversión alimenticia se determinó dividiendo el consumo del alimento entre la ganancia de peso. El índice de eficiencia europeo se obtuvo mediante la fórmula (Viabilidad x Ganancia diaria de peso x 100) / Índice de conversión alimenticia.

A los 28 días de edad, se sacrificaron cuatro pollos de cada unidad experimental en la sala de beneficio de la granja de aves de la UNALM. Se retiraron las tibias izquierdas y fueron introducidas en mallas mosquiteras e identificadas individualmente. Dicho envoltorio fue sometido a agua hirviendo por $15 \mathrm{~min}$ para remover el tejido del hueso (Applegate y Lilburn, 2002), procedimiento que no altera el contenido mineral ni la densidad del hueso y permite retirar el $80 \%$ de la grasa contenida en los huesos (Almeida y Bruno, 2008). Los tejidos y cartílagos fueron retirados manualmente siguiendo el procedimiento de Baumel y Witmer (1993). Los huesos fueron limpiados con papel toalla y colocados en un recipiente plástico. Una semana después se hicieron las mediciones respectivas. 
Cuadro 1. Composición, valores nutricionales calculados y nutrientes de las dietas normal y deficiente en fósforo

\begin{tabular}{lcc}
\hline & \multicolumn{2}{c}{ Dieta } \\
\cline { 2 - 3 } Ingrediente & Normal en P & Deficiente en P \\
\hline Maíz amarillo molido & 61.160 & 61.887 \\
Torta de soya & 31.471 & 31.319 \\
Aceite crudo de soya & 3.638 & 3.409 \\
Fosfato dicálcico & 1.822 & 0.723 \\
Carbonato de calcio & 0.611 & 1.364 \\
DL-Metionina & 0.203 & 0.202 \\
Lisina-HCL & 0.137 & 0.140 \\
Cloruro de colina 60 & 0.100 & 0.100 \\
Premezcla vitamina-mineral & 0.100 & 0.100 \\
Secuestrante & 0.100 & 0.100 \\
Antihongos & 0.050 & 0.050 \\
Antioxidante & 0.050 & 0.050 \\
Antibiótico & 0.050 & 0.050 \\
Sal común & 0.457 & 0.457 \\
Coccidiostato & 0.050 & 0.050 \\
\hline Total & 100.00 & 100.00 \\
\hline Valor nutricional (calculado) & & 3035 \\
E.M., Kcal/kg & 3035 & 20.50 \\
Proteína total, \% & 20.50 & 1.18 \\
Lisina total, \% & 1.18 & 0.87 \\
Met + Cist total, \% & 0.87 & 0.80 \\
Calcio, \% & 0.80 & 0.25 \\
Fósforo disponible, \% & 0.45 & \\
Nutrientes analizados ${ }^{1}$ & & 0.59 \\
Proteína total, \% & 19.55 & \\
Fósforo total, \% & 0.76 & \\
\hline
\end{tabular}

${ }^{1}$ Laboratorio de Evaluación Nutricional de Alimentos (LENA) - UNALM

Se determinó la densidad ósea (peso/ volumen) (diámetro de la diáfisis, largo, volumen y peso) y los indicadores de mineralización de la tibia que fueron: densidad ósea (Rath et al., 2000), índice de Seedor (Seedor et al., (1991), índice de Quetelet (Martinez, 2012; Rutten et al., 2002), índice de robusticidad (Kokabagli, 2001; Mutus et al., 2006; Martínez, 2012) y porcentaje de cenizas (García y Dale, 2006). Para medir el peso de la tibia (mg) se empleó una balanza analítica con una aproximación de $0.1 \mathrm{mg}$ y para la determinación de las cenizas se utilizó un horno mufla. El porcentaje de pierna se determinó con base a la relación porcentual entre el peso vivo y el peso de las piernas. 
Las densidades óseas del fémur, tibia y tarso de las aves se determinaron mediante la volumetría del hueso (Quarantelli et al., 2007), considerándose como densidad ósea a la masa de material orgánico e inorgánico en el hueso por unidad de volumen del mismo (Rath et al., 2000). Se calculó dividiendo el peso del hueso $(\mathrm{mg})$ entre el volumen $\left(\mathrm{cm}^{3}\right)$. Para la determinación del volumen de los huesos se emplearon probetas graduadas con capacidad de 10 y $25 \mathrm{~cm}^{3}$ y con una aproximación de $0.2 \mathrm{~cm}^{3}$. El largo $(\mathrm{mm})$, diámetro de diáfisis $(\mathrm{mm})$ e índice de forma $(\mathrm{mm})$ de las tibias fueron medidos empleando un vernier Uyustools profesional con una capacidad para $15 \mathrm{~cm}$ y con una aproximación de $0.05 \mathrm{~mm}$.

El índice de Seedor se determinó dividiendo el peso del hueso (mg) entre su largo $(\mathrm{mm})$. El Índice de masa corporal o de Quetelet se obtuvo dividiendo el peso del hueso (mg) entre la longitud al cuadrado del mismo $\left(\mathrm{mm}^{2}\right)$. El índice de robusticidad se obtuvo dividiendo el largo del hueso $(\mathrm{mm})$ entre el peso $(\mathrm{g})$ elevado a la potencia $1 / 3$.

Se empleó un diseño completamente al azar con seis tratamientos y cinco repeticiones por tratamiento. El análisis de varianza se llevó a cabo usando el programa Statistical Analysis System (SAS, 2000). La comparación entre medias se determinó mediante la Prueba de Duncan.

\section{Resultados y Discusión}

En el Cuadro 2 se muestra el peso vivo inicial y final (g/ave) y las ganancias de peso (g/ave) obtenidos a los 28 días de edad en los seis tratamientos. La mayor ganancia de peso se obtuvo en $\mathrm{T} 1(0.45 \%$ de $\mathrm{P})$, en comparación con $\mathrm{T} 2(0.25 \%$ de $\mathrm{P})$ y el resto de tratamientos $(\mathrm{p}<0.05)$. En los tratamientos con fitasas, la fitasa A (T3) registró la mejor ganancia de peso $(1297 \mathrm{~g})$ seguidos por $\mathrm{T} 5, \mathrm{~T} 4$ y T6. No hubo diferencia estadística entre los promedios obtenidos con $\mathrm{T} 3$ y $\mathrm{T} 5$ ni entre
T4 y T6, pero T3 y T5 difieren con respecto a T6 $(\mathrm{p}<0.05)$.

Las diferencias de rendimiento encontradas en este estudio se deben probablemente a que la formulación de las dietas se basó en la matriz de cada fitasa comercial, considerando solo al fósforo disponible, más no al resto de nutrientes que la constituyen; vale decir, proteína cruda, aminoácidos, calcio y energía metabolizable. Asimismo, otro factor a considerar es el origen de la fitasa (fitasa A: Aspergillus niger; fitasas $\mathrm{B}$ y $\mathrm{D}$ : Escherichia coli; y fitasa C: mezcla de enzimas vegetales), donde cada una de ellas hidroliza al ácido fítico en distintas posiciones (C3 o C6).

Los resultados fueron similares a los encontrados por Godoy et al. (2002), quienes, a los 28 días experimentales, reportaron ganancias máximas de $1232 \mathrm{~g}$, siendo necesaria la adición de $540 \mathrm{U} / \mathrm{kg}$ para la liberación de un gramo de fósforo con un nivel de $0.25 \%$ de fósforo disponible en la dieta. En el presente estudio, las fitasas A y C, con 10000 y $5000 \mathrm{U} / \mathrm{kg}$, respectivamente, lograron incrementos de pesos ligeramente superiores (1297 y 1266, respectivamente) sin llegar a ser similares a la dieta normal en fósforo (T1).

El consumo total de alimento (g/ave) y la conversión alimenticia acumulada a $\operatorname{los} 28$ días de edad por tratamiento se presentan en el Cuadro 2. Los mayores consumos de alimento se registraron para $\mathrm{T} 1, \mathrm{~T} 3$ y $\mathrm{T} 5$, en tanto que niveles deficientes de P (T2) mostraron el menor consumo $(1603 \mathrm{~g})(\mathrm{p}<0.05)$. El consumo de alimento con fitasas fueron superiores al consumo de $\mathrm{T} 2$, siendo $\mathrm{T} 3$ y $\mathrm{T} 5$ superiores respecto a T4 y T6 $(\mathrm{p}<0.05)$. Consumos similares fueron reportados por Assuena et al. (2009) y Hatsumi et al. (2008) en estudios de 21 días, donde agregaron fitasas a niveles de 250 y $1000 \mathrm{U} / \mathrm{kg}$ y diferentes valores de fósforo $(0.48$ y $0.27 \%$ de $\mathrm{Pd})$, respectivamente. El mayor consumo de alimento en dietas bajas en fósforo, pero con la adición de fitasas, concuerda con los hallazgos de Hussein (2006). 
Cuadro 2. Comportamiento productivo de pollos de carne alimentados con dietas bajas en fósforo disponible y suplementadas con diferentes fuentes de fitasa (Periodo 1 a 28 días de edad)

\begin{tabular}{lcccccc}
\hline Medición $^{1}$ & T1 $^{2}$ & T2 & T3 & T4 & T5 & T6 \\
\hline Peso vivo inicial, g/ave & $45^{\mathrm{a}}$ & $45^{\mathrm{a}}$ & $44^{\mathrm{a}}$ & $45^{\mathrm{a}}$ & $45^{\mathrm{a}}$ & $44^{\mathrm{a}}$ \\
Peso vivo final, g/ave & $1419^{\mathrm{a}}$ & $896^{\mathrm{e}}$ & $1340^{\mathrm{b}}$ & $1238^{\mathrm{cd}}$ & $1311^{\mathrm{cb}}$ & $1177^{\mathrm{d}}$ \\
Ganancia de peso, g/ave & $1374^{\mathrm{a}}$ & $851^{\mathrm{e}}$ & $1297^{\mathrm{b}}$ & $1193^{\mathrm{cd}}$ & $1266^{\mathrm{cb}}$ & $1133^{\mathrm{d}}$ \\
Ganancia de peso, g/día & $49.1^{\mathrm{a}}$ & $30.4^{\mathrm{e}}$ & $46.3^{\mathrm{b}}$ & $42.6^{\mathrm{cd}}$ & $45.2^{\mathrm{cb}}$ & $40.5^{\mathrm{d}}$ \\
Consumo de alimento, g/ave & $1919^{\mathrm{a}}$ & $1603^{\mathrm{c}}$ & $1846^{\mathrm{ab}}$ & $1747^{\mathrm{b}}$ & $1812^{\mathrm{ab}}$ & $1776^{\mathrm{b}}$ \\
Conversión alimenticia, g/g & $1.40^{\mathrm{c}}$ & $1.89^{\mathrm{a}}$ & $1.43^{\mathrm{c}}$ & $1.47^{\mathrm{cb}}$ & $1.43^{\mathrm{cb}}$ & $1.57^{\mathrm{b}}$ \\
Mortalidad, \% & 4.0 & 38.5 & 0 & 10.0 & 4.0 & 10.0 \\
IEPE $^{3}$ & 348 & 104 & 336 & 272 & 314 & 241 \\
\hline
\end{tabular}

$a, b, c, d, e$ Superíndices diferentes dentro de filas indican diferencia estadística $(p<0.05)$

${ }^{1} \mathrm{n}=50$ aves por tratamiento

2 Tratamientos: T1: DNP (0.45\% Pd); T2: DDP (0.25\% Pd); T3: DDP + Fitasa A (100 g/t); T4: DDP +

Fitasa B (100 g/t); T5: DDP + Fitasa C (50 g/t); T6: DDP + Fitasa D (50 g/t)

2 Índice de Eficiencia Productivo Europeo

Cuadro 3. Rendimiento de carcasa y piernas de pollos de carne de 28 días de edad alimentados con dietas bajas en fósforo disponible y suplementadas con cuatro fuentes de fitasas

\begin{tabular}{lcccccc}
\hline Medición & T1 & T2 & T3 & T4 & T5 & T6 \\
\hline Peso carcasa, g & $1140^{\mathrm{a}}$ & $713^{\mathrm{d}}$ & $1053^{\mathrm{b}}$ & $1011^{\mathrm{bc}}$ & $1043^{\mathrm{b}}$ & $967^{\mathrm{c}}$ \\
Rendimiento carcasa, \% & $82.6^{\mathrm{a}}$ & $75.7^{\mathrm{c}}$ & $81.6^{\mathrm{ab}}$ & $80.1^{\mathrm{b}}$ & $80.5^{\mathrm{ab}}$ & $80.8^{\mathrm{ab}}$ \\
Peso de piernas, g & $328^{\mathrm{a}}$ & $207^{\mathrm{d}}$ & $310^{\mathrm{b}}$ & $294^{\mathrm{bc}}$ & $300^{\mathrm{b}}$ & $280^{\mathrm{c}}$ \\
Piernas, \% & $28.8^{\mathrm{a}}$ & $29.1^{\mathrm{a}}$ & $29.4^{\mathrm{a}}$ & $29.0^{\mathrm{a}}$ & $28.8^{\mathrm{a}}$ & $29.0^{\mathrm{a}}$ \\
\hline
\end{tabular}

$a, b, c, d$ Superíndices diferentes dentro de filas indican diferencia estadística $(p<0.05)$

${ }^{1}$ Tratamientos: T1: DNP (0.45\% Pd); T2: DDP (0.25\% Pd); T3: DDP + Fitasa A (100 g/t); T4: DDP +

Fitasa B (100 g/t); T5: DDP + Fitasa C (50 g/t); T6: DDP + Fitasa D (50 g/t)

La mejor conversión alimenticia fue registrada para T1, T3, T4 y T5, en tanto que $\mathrm{T} 2$ registró la conversión menos eficiente $(p<0.05)$. Asimismo, entre las fitasas testadas, la fitasa A(T3) fue similar con las fitasas B y C (T4 y T5, respectivamente), pero diferente a la fitasa D $(\mathrm{T} 6)(\mathrm{p}<0.05)$. Hatsumi et al. (2008) reportaron una conversión de 1.39 a los 21 días. Por su parte Godoy et al. (2002) encontraron conversiones ligeramente superiores (1.28) a los 28 días, mientras que Ahmad (2008) obtuvo conversiones menos eficientes (1.52) a los 28 días, y Assuena et al. (2009), Oliveira et al. (2009) y Karimi et al. (2011) obtuvieron conversiones de 1.44, 1.57 y 2.21 , respectivamente, a los 21 días. 
Cuadro 4. Características de tibias de pollos de carne de 28 días de edad alimentados con dietas bajas en fósforo disponible y suplementadas con cuatro fuentes de fitasas

\begin{tabular}{lccc}
\hline Tratamiento & Peso $(\mathrm{mg})$ & Ceniza $(\mathrm{mg})$ & Ceniza (\%) \\
\hline T1: DNP $(0.45 \% \mathrm{Pd})$ & $3286^{\mathrm{a}}$ & $1459^{\mathrm{a}}$ & $44.4^{\mathrm{a}}$ \\
T2: DDP $(0.25 \% \mathrm{Pd})$ & $1973^{\mathrm{d}}$ & $820^{\mathrm{d}}$ & $41.6^{\mathrm{c}}$ \\
T3: DDP + Fitasa A (100 g/t) & $3070^{\mathrm{b}}$ & $1321^{\mathrm{b}}$ & $43.0^{\mathrm{abc}}$ \\
T4: DDP + Fitasa B (100 g/t) & $2896^{\mathrm{b}}$ & $1230^{\mathrm{b}}$ & $42.5^{\mathrm{bc}}$ \\
T5: DDP + Fitasa C (50 g/t) & $2872^{\mathrm{b}}$ & $1243^{\mathrm{b}}$ & $43.3^{\mathrm{abc}}$ \\
T6: DDP + Fitasa D (50 g/t) & $2583^{\mathrm{c}}$ & $1134^{\mathrm{c}}$ & $43.9^{\mathrm{ab}}$ \\
\hline
\end{tabular}

$a, b, c$ Superíndices diferentes dentro de columnas indican diferencia estadística $(p<0.05)$

El mayor porcentaje de mortalidad lo registró T2 $(38.5 \%)$, seguido por T4 y T6 $(10 \%)$, T5 у T1 (4\%) у T3 (0\%) (Cuadro 2$)$. Estos resultados se asemejan a los reportados por Karimi et al. (2011), quienes registraron $9.5 \%$ de mortalidad con dietas bajas en fósforo $(0.20 \%)$ y con la adición de fitasas (500 FTU).

T1 logró el mejor Índice de Eficiencia Productivo Europeo seguido de T3 y T5 (fitasas A y C) logrando índices superiores de 300 considerado dentro del rango de una producción eficiente, según Aguavil (2012). Por otro lado, las aves de T2, T4 y T6 no alcanzaron el valor aceptable de rentabilidad (Cuadro 2).

El Cuadro 3 muestra el rendimiento de carcasa, peso vivo, peso de piernas, peso de carcasa y porcentaje de piernas de los cuatro animales seleccionados por unidad experimental. El rendimiento de carcasa encontrado usando fitasas comerciales no presenta diferencias estadísticas con respecto al nivel normal (T1), a excepción de T4 (fitasa B). T2 fue menor y estadísticamente diferente $(\mathrm{p}<0.05)$ al resto de tratamientos. Este ensayo reporta mejores rendimientos de carcasa (81.6\%) en comparación con los estudios de Ahmad (2008) y Oliveira et al. (2009), quiénes obtuvieron 72 y $78 \%$, respec- tivamente, como resultado de una mejor conversión alimenticia.

El peso de las piernas en T1 superó a todos los tratamientos $(\mathrm{p}<0.05)$. Por otro lado, los pesos en T3, T4 y T5 fueron superiores a T2 y T6, excepto T4 que fue similar a T6 $(p<0.05)$. El porcentaje de piernas fue similar entre tratamientos.

Los resultados de las características de la tibia (en base seca) de los pollos se presentan en el Cuadro 4. El peso de tibia de T1 fue mayor y estadísticamente diferente al resto de los tratamientos $(\mathrm{p}<0.05)$. T3, T4 y T5 fueron similares entre sí, pero diferentes a T6 $(p<0.05)$, mientras que T2 presentó el menor peso de tibia $(p<0.05)$. Similares resultados se observaron para el peso de las cenizas.

Los porcentajes de cenizas fueron relativamente similares entre tratamientos, habiendo diferencia significativa entre $\mathrm{T} 1$ y $\mathrm{T} 2$ $(p<0.05)$. Resultados similares fueron reportados por Kocabagli (2001) y Panda et al. (2007), con 300 y 500 FTU de fitasas con dietas bajas en fósforo, logrando rendimientos de $44.1 \%$ y $44.92 \%$, respectivamente a los 21 días. Los resultados indican que existe una mejora en la liberación del fósforo fítico del alimento, causado por las fitasas, refleja- 
Cuadro 5. Características morfométricas de las tibias de pollos de carne de 28 días de edad alimentados con dietas bajas en fósforo disponible y suplementadas con cuatro fuentes de fitasas

\begin{tabular}{lcccccc}
\hline Medición & $\mathrm{T} 1^{1}$ & $\mathrm{~T} 2$ & $\mathrm{~T} 3$ & $\mathrm{~T} 4$ & $\mathrm{~T} 5$ & $\mathrm{~T} 6$ \\
\hline Peso, mg & $3787^{\mathrm{bc}}$ & $2777^{\mathrm{d}}$ & $3599^{\mathrm{c}}$ & $4165^{\mathrm{a}}$ & $3975^{\mathrm{ab}}$ & $3705^{\mathrm{bc}}$ \\
Largo, mm & $78.22^{\mathrm{a}}$ & $61.02^{\mathrm{c}}$ & $77.01^{\mathrm{ab}}$ & $71.32^{\mathrm{b}}$ & $75.46^{\mathrm{ab}}$ & $72.94^{\mathrm{ab}}$ \\
Diámetro de diáfisis, mm & $6.71^{\mathrm{ab}}$ & $6.16^{\mathrm{b}}$ & $6.63^{\mathrm{ab}}$ & $6.55^{\mathrm{ab}}$ & $6.75^{\mathrm{a}}$ & $6.45^{\mathrm{ab}}$ \\
Volumen, cm & $4.68^{\mathrm{a}}$ & $2.51^{\mathrm{d}}$ & $4.43^{\mathrm{a}}$ & $3.17^{\mathrm{c}}$ & $3.70^{\mathrm{b}}$ & $3.43^{\mathrm{bc}}$ \\
Índice de forma, mm & $11.70^{\mathrm{a}}$ & $9.84^{\mathrm{b}}$ & $11.66^{\mathrm{a}}$ & $10.85^{\mathrm{a}}$ & $11.25^{\mathrm{a}}$ & $11.34^{\mathrm{a}}$ \\
\hline
\end{tabular}

a,b,c,d Superíndices diferentes dentro de filas indican diferencia estadística $(p<0.05)$

${ }^{1}$ Tratamientos: T1: DNP (0.45\% Pd); T2: DDP (0.25\% Pd); T3: DDP + Fitasa A (100 g/t); T4: DDP +

Fitasa B (100 g/t); T5: DDP + Fitasa C (50 g/t); T6: DDP + Fitasa D (50 g/t)

Cuadro 6. Respuesta del grado de mineralización de las tibias de pollos de carne de 28 días de edad alimentados con dietas bajas en fósforo disponible y suplementadas con cuatro fuentes de fitasas

\begin{tabular}{lcccccc}
\hline Medición & $\mathrm{T} 1^{1}$ & $\mathrm{~T} 2$ & $\mathrm{~T} 3$ & $\mathrm{~T} 4$ & $\mathrm{~T} 5$ & $\mathrm{~T} 6$ \\
\hline Densidad, $\mathrm{mg} / \mathrm{cm}^{3}$ & $815^{\mathrm{c}}$ & $1152^{\mathrm{b}}$ & $816^{\mathrm{c}}$ & $1511^{\mathrm{a}}$ & $1092^{\mathrm{b}}$ & $1111^{\mathrm{b}}$ \\
Índice de Seedor, $\mathrm{mg} / \mathrm{mm}$ & $48.40^{\mathrm{bcd}}$ & $44.64^{\mathrm{d}}$ & $46.65^{\mathrm{cd}}$ & $56.23^{\mathrm{a}}$ & $52.59^{\mathrm{ab}}$ & $50.74^{\mathrm{bc}}$ \\
Índice de Quetelet, $\mathrm{mg} / \mathrm{mm}^{2}$ & $0.62^{\mathrm{a}}$ & $0.89^{\mathrm{a}}$ & $0.61^{\mathrm{a}}$ & $1.04^{\mathrm{a}}$ & $0.70^{\mathrm{a}}$ & $0.70^{\mathrm{a}}$ \\
Índice de robusticidad, mm & $5.02^{\mathrm{a}}$ & $4.47^{\mathrm{c}}$ & $5.03^{\mathrm{a}}$ & $4.64^{\mathrm{bc}}$ & $4.78^{\mathrm{b}}$ & $4.73^{\mathrm{b}}$ \\
\hline
\end{tabular}

$a, b, c, d$ Superíndices diferentes dentro de filas indican diferencia estadística $(p<0.05)$

${ }^{1}$ Tratamientos: T1: DNP (0.45\% Pd); T2: DDP (0.25\% Pd); T3: DDP + Fitasa A (100 g/t); T4: DDP +

Fitasa B (100 g/t); T5: DDP + Fitasa C (50 g/t); T6: DDP + Fitasa D (50 g/t)

do en el almacenamiento en tejidos óseos (García y Dale 2006). Uculmana (2015) demostró que el fósforo, además del calcio, es el mineral que determina la cantidad de ceniza almacenada en el hueso.

En el Cuadro 5 se muestran los resultados del peso $(\mathrm{mg})$, largo $(\mathrm{mm})$, diámetro de diáfisis $(\mathrm{mm})$, volumen $\left(\mathrm{cm}^{3}\right)$ e índice de for$\mathrm{ma}(\mathrm{mm})$ de las tibias de las aves. La adición de la enzima fitasa B en T4 a dietas deficientes en fósforo mejoró significativamente $(p<0.05)$ el peso del hueso en relación a la dieta T1 con niveles normales de fósforo. Kocabagli (2001) encontró resultados mayores a los 21 días, donde $300 \mathrm{U} / \mathrm{kg}$ en $0.26 \%$ Pd fueron necesarios para lograr $6599 \mathrm{mg}$ de peso.

El largo de la tibia fue menor en T2 $(p<0.05)$, no habiendo diferencias significativas en los tratamientos deficientes de fósforo con adición de fitasas. Estos valores son inferiores a lo reportados por Kocabagli 
(2001) y Mondal et al. (2007), quienes encontraron largos de 92 y $123 \mathrm{~mm}$ (700 y 500 U de fitasas, respectivamente) a los 21 días de edad.

El diámetro de diáfisis tibial fue similar entre tratamientos, excepto para T2, el cual resultó estadísticamente menor a T5. Por otro lado, los volúmenes variaron significativamente entre tratamientos $(p<0.05)$. En el índice de forma se puede apreciar el efecto positivo que tuvo la adición de las enzimas fitasas, no encontrándose ninguna diferencia entre los tratamientos con fitasas y $\mathrm{T} 1$, pero una diferencia significativa con $\mathrm{T} 2(\mathrm{p}<0.05)$.

En el Cuadro 6 se observa la respuesta del grado de mineralización de las tibias expresados en densidad $\left(\mathrm{mg} / \mathrm{cm}^{3}\right)$, índice de Seedor $(\mathrm{mg} / \mathrm{mm})$, índice de Quetelet $(\mathrm{mg} /$ $\mathrm{mm}^{2}$ ) e índice de robusticidad (mm). La mayor fortaleza ósea (densidad) se observó en $\mathrm{T} 4$ (fitasa B) y la menor en T1 y T3 $(\mathrm{p}<0.05)$. Rath et al. (2000) demostraron que la densidad ósea es un indicador de la fortaleza del hueso y refleja el contenido mineral del hueso, por lo cual una menor densidad ósea aumenta el riesgo de fracturas. El hallazgo de una densidad ósea disminuida en T1 con respecto al resto de tratamientos podría deberse, según Rath et al. (2000) a una desmineralización ósea provocada por otros factores diferentes al nutricional.

En el índice de Seedor, T4 (fitasa B) obtuvo mayores valores que los demás tratamientos $(p<0.05)$, mientras que en el índice de Quetelet no se encontraron diferencias estadísticas entre tratamientos. El índice de robusticidad indica que el hueso es más fuerte cuanto menor es este parámetro (Monteagudo et al., 1997) y, en este caso, T2 y T4 logran ser significativamente mejores al resto de tratamientos con excepción de $\mathrm{T} 5$ y T6 que son similares a T4, pero no a $\mathrm{T} 2$.

\section{Conclusiones}

- Las respuestas productivas de las aves que recibieron la dieta deficiente en fósforo con la adición de fitasas fueron menores que el de las aves alimentadas con la dieta normal en fósforo, pero mayores que el de las aves con dieta deficiente de fósforo.

- La menor respuesta productiva e integridad ósea correspondió al grupo de aves alimentadas con la dieta deficiente en fósforo.

- Los mayores pesos, en base seca, de la tibia y cenizas correspondieron a las aves alimentadas con la dieta normal en fósforo.

\section{Literatura Citada}

1. Aguavil J. 2012. Evaluación del efecto de un probiótico nativo elaborado en base a Lactobacillus acidophilus y Bacillus subtilis sobre el sistema gastrointestinal en pollos broiler ROSS-308 en Santo Domingo de los Tsáchilas. Tesis de Ingeniero Agropecuario. Santo Domingo, Ecuador: Escuela Politécnica del Ejército. 90 p. [Internet]. Disponible en: http:/ /repositorio.espe.edu.ec/bitstream/ 21000/5213/1/T-ESPE-IASA\%20II\%20$\% 20002399 . p d f$

2. Ahmad RI. 2008. Effects of different level of phytase on broilers performance and body status of phosphorus. MSc Thesis. Nablus, Palestine: An Najah National University. $70 \mathrm{p}$.

3. Angel R, Saylor WW, Mitchell AD, Powers W, Applegate TJ. 2006. Effect of dietary phosphorus, phytase, and 25hydroxycholecalciferol on broiler chicken bone mineralization, litter phosphorus, and processing yields. Poult Sci 85: 12001211. doi: $10.1093 / \mathrm{ps} / 85.7 .1200$ 
4. Almeida P, Bruno LDG. 2008. Bone mineral density: review. Rev Bras Cienc Avic 8: 69-73. doi: 10.1590/S1516635X2006000200001

5. Applegate TJ, Lilburn MS. 2002. Growth of the femur and tibia of a commercial broiler line. Poult Sci 81: 1289-1294.

6. Applegate TJ, Joern BC, NussbaumWagler DL, Angel R. 2003. Water-soluble phosphorus in fresh broiler litter is dependent upon phosphorus concentration fed but not on fungal phytase supplementation. Poult Sci 82: 10241029. doi: $10.1093 / \mathrm{ps} / 82.6 .1024$

7. Assuena V, Junqueira, OM, Duarte KF, Laurentiz AC, Filardi RS, Sgavioli S. 2009. Effect of dietary phytase supplementation on the performance, bone densitometry, and phosphorus and nitrogen excretion of broilers. Rev Bras Cienc Avic 11: 25-30. doi: 10.1590/ S1516-635X2009000100005

8. Baumel JJ, Witmer LM. 1953. Osteología. In: Baumel JJ, King AS, Breazile GE, Evans HE, Vanden Berge JC (eds). Handbook of avian anatomy: nomina anatomica avium. $2^{\text {nd }}$ ed. Cambridge, UK: Nuttall Ornithological Club. $779 \mathrm{p}$.

9. Cobb-Vantres. 2012. Guía de manejo del pollo de engorde. [Internet]. Disponible en: http://cobb-vantress.com/languages/ guidefiles/b5043b0f-792a-448e-b4a14aff9a30e9eb_es.pdf

10. García AR, Dale NM. 2006. Foot ash as a means of quantifying bone mineralization in chicks. J Appl Poult Res 15: 103-109. doi: https://doi.org/10.1093/ japr/15.1.103

11. Godoy S, Hernandez G, Chicco C. 2002. Efecto de la suplementación de fitasa microbial en la utilización de fósforo fítico en pollos de engorde alimentados con dietas de maíz-soya. Rev Cient Luz 12(Supl 2). 519-523.

12. Harland BF, Morris ER. 1995. Phytate: a good or a bad food component. Nutrit Res 15: 733-754. doi: 10.1016/ 0271-5317(95)00040-P
13. Hatsumi FE, Kazue SN, Barros DLR; Neme R, Kochenborger FJB, Márcia MS. 2008. Efeito da suplementação de fitase sobre o desempenho e a digestibilidade dos nutrientes em frangos de corte. R Bras Zootec 37: 629-635. doi: 10.1590/S1516-35982008000400007

14. Hussein AS. 2006. The influence of phytase enzyme supplementation on performance and calcium and phosphorus metabolism of broiler chicks. In: $12^{\text {th }}$ European Poultry Conference. Verona, Italy.

15. Karimi A, Bedford MR, Sadegui GH, Ghobadi Z. 2011. Influence of dietary non-phytate phosphorous levels and phytase supplementation on the performance and bone characteristics of broilers. Rev Bras Cienc Avic 13: 4351. doi: 10.1590/S1516-635X2011000100007

16. Kocabagli N. 2001. The effect of dietary phytase supplementation at different levels on tibial bone characteristics and strength in broilers. Turk J Vet Anim Sci 25: 797-802.

17. Martínez DA. 2012. Evaluación de un producto a base de aceite esencial de orégano sobre la integridad intestinal, la capacidad de absorción de nutrientes y el comportamiento productivo de pollos de carne. Tesis de Magíster. Lima: Univ Nacional Agraria La Molina. 368 p.

18. Mondal MK, Panda S, Biswas P. 2007. Effect of microbial phytase in soybean meal based broiler diets containing low phosphorous. Int J Poult Sc1 6: 201-206. doi: 10.3923/ijps.2007.201.206

19. Monteagudo MD, Hernandez ER, Seco C, Gonzales-Riola J, Revilla M, Villa LF, Rico H. 1997. Comparison of the bone rousticity index and bone weight/ bone length index with the results of bone densitometry and bone histomorphometry in experimental estudies. Acta Anat 160: 195-199.

20. Mutus R, Kocabagli N, Alp M, Acar $N$, Eren M, Gezen SS. 2006. The effect of dietary probiotic supplementation on tibial bone characteristics and 
strength in broiler. Poult Sci 85: 1621 1625.

21. Nair VC, Duvnjak Z. 1991. Phytic acid content reduction in canola meal by various microorganisms in a solid state fermentation process. Acta Biotechnol 11:211-218. doi: 10.1002/abio.370110306

22. Oliveira MC, Marquez RE, Gravena $R A$, Traldi AB, Godoy CR, Barbosa de Moraes VM. 2009. Fitase em dietas com níveis reduzidos de fósforo não-fítico para frangos de corte. Biotemas 22: 169176.

23. Panda AK, Rao SVR, Raju VLN, Gajula SS, Branja SK. 2007. Performance of broiler chickens fed low non phytate phosphorus diets supplemented with microbial phytase. J Poult Sci 44: 258-264.

24. Penn CJ, Mullins GL, Zelazny LW, Warren JG, McGrath JM. 2004. Surface runoff losses of phosphorus from Virginia soils amended with turkey manure using phytase and high available phosphorus corn diets. J Environ Qual 33: 143-1439.

25. Quarantelli A, Cacchioli A, Romanelli S, Righi F, Alpigiani I, Gabbi C. 2007. Effects of different levels of dietary biotin on the performance and bone structure of broiler. Ital J Anim Sci 6: 57. doi: 10.4081/ijas.2007.5

26. Rath NC, Huff GR, Huff WE, Balog JM. 2000. Factors regulating bone maturity and strength in poultry. Poult Sci 79: 1024-1032.
27. Rutten M, Leterrier C, Constantin P, Reiter K, Bessel W. 2002. Bone development and activity in chickens in response to reduced weight-load on legs. Anim Res 51: 327-336. doi: 10.1051/ animres: 2002027

28. SAS Institute. 2000. The SAS System for Windows. User's Guide Statistics v 8. SAS Institute, Inc., Cary, USA.

29. Seedor JG, Quartuccio HA, Thompson DD. 1991. The bisphosphonate alendronate (MK-217) inhibits bone loss due to ovariectomy in rats. J Bone Miner Res 6: 339-346. doi: 10.1002/jbmr.5650060405

30. Shaw AL, Blake JP, Gordon RW. 2010. Evaluation of commercial phytase enzymes on performance and tibiabreaking strength of male broiler chicks. J Appl Poult Res 19: 415-421. doi: 10.3382/japr.2009-00121

31. Uculmana C. 2015. Efecto de la relación calcio : fósforo disponible sobre el crecimiento alométrico, morfometría, integridad y mineralización ósea en pollos de engorde. Tesis de Ingeniero Zootecnista. Lima, Perú: Univ Nacional Agraria La Molina. $76 \mathrm{p}$

32. Woyengo TA, Guenter W, Sands JS, Nyachoti CM, Mirza MA. 2010. Nutrient utilization and performance responses of broilers fed a wheat-based diet supplemented with phytase and xylanase alone or in combination. Anim Feed Sci Technol 146: 113-123. doi: 10.1016/j.anifeedsci.2007.11.013 